\title{
Ion polarization control in MEIC rings using small magnetic field integrals
}

\author{
Ya.S. Derbenev, F. Lin, V.S. Morozov ${ }^{1}$, Y. Zhang \\ Thomas Jefferson National Accelerator Facility, Newport News, Virginia 23606, USA \\ E-mail: derbenev@jlab.org, fanglei@jlab.org, morozov@jlab.org, \\ yzhang@jlab.org
}

\section{A.M. Kondratenko, M.A. Kondratenko}

Science and Technique Laboratory „Zaryad“, Novosibirsk 630058, Russia

E-mail: kondratenkom@mail.ru

\section{Yu.N. Filatov}

Moscow Institute of Physics and Technology, Dolgoprudny 141700, Russia Joint Institute for Nuclear Research, Dubna 141980, Russia

E-mail: yuriifilatoff@mail.ru

The Electron-Ion Collider (EIC) proposed by Jefferson Lab is designed to provide high polarization of both colliding beams. In comparison to conventional circular colliders, its figure 8 shaped rings provide a unique capability to control the polarization of any particle species including deuterons by using small magnetic field integrals. This expands the experimental opportunities at EIC. We present schemes for preserving the particle polarization during acceleration in the pre-booster and large booster. We also present schemes for controlling the ion polarization up to the energy of $100 \mathrm{GeV}$ in the collider ring that allow one to adjust the beam polarization in any orientation in its experimental straights. The deuteron polarization is controlled by weak solenoids while the proton polarization is controlled by weak radial fields. The components of these schemes are optically transparent to the orbital motion when integrated into the magnetic structure of the rings. We use the spin response function formalism to evaluate the zeroth harmonic of the spin perturbation and investigate the possibility of its compensation. For control of the proton polarization in the high-energy stage at energies above $100 \mathrm{GeV}$, we plan to use the conventional approach of Siberian snakes and spin rotators.

XVth International Workshop on Polarized Sources, Targets, and Polarimetry

September 9-13, 2013

Charlottesville, Virginia, USA

1

Speaker 


\section{Introduction}

Proton beam's polarization in a "figure- 8 " collider can be controlled using schemes with Siberian snakes and rotators analogous to those in conventional circular accelerators $[1,2]$. Siberian snakes provide a spin reversal in their insertion section and require magnetic fields with a large integrated strength ("strong" fields). In such schemes, figure- 8 accelerators and conventional accelerators become equivalent in terms of using additional spin control devices. However, this does not make use of the unique feature of the figure- 8 ring itself, namely, the spin tune being zero, independent of the beam energy. In particular, using schemes with Siberian snakes, it is not practical to control the deuteron polarization. The figure- 8 ring structure opens an additional unique capability of efficiently controlling the deuteron and proton polarization by "small" solenoids and dipoles.

A schematic of Jefferson Lab's proposed Medium-energy Electron-Ion Collider (MEIC) complex [1] is shown in figure 1. The ion acceleration scheme is illustrated in figure 2. Polarized ions produced by an ion source are first accelerated to $285 \mathrm{MeV}$ by an SRF linac. The beam is then accelerated to $3 \mathrm{GeV}$ in the prebooster, transferred into the large booster for further acceleration to $20 \mathrm{GeV} / \mathrm{c}$ and finally injected into the collider ring for acceleration to an experimental energy of up to $100 \mathrm{GeV} / \mathrm{c}$. In the full-energy Electron-Ion Collider (EIC) scenario, the medium-energy collider ring will be used as another booster for delivering a $100 \mathrm{GeV} / \mathrm{c}$ beam into the full-energy collider ring.

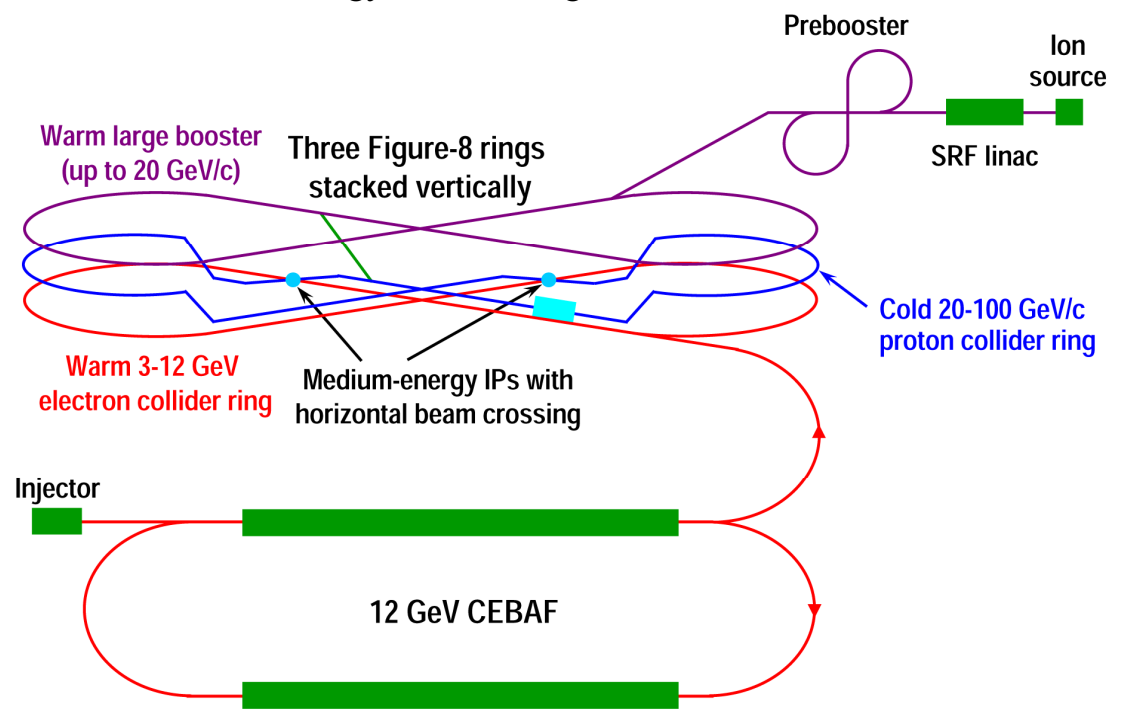

Figure 1: MEIC schematic.

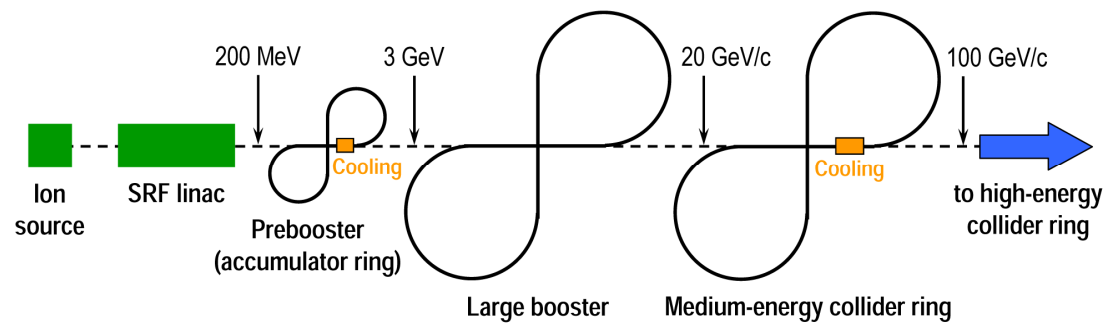

Figure 2: Ion acceleration scheme in MEIC. 


\section{Preservation of the polarization during particle acceleration in the prebooster and large booster}

Use of a "small" longitudinal field at MEIC solves the problem of polarization preservation for deuterons and protons during the whole acceleration cycle including the linac, prebooster, large booster and collider ring. It can be easily seen that, to attain longitudinal polarization in a straight section, it is sufficient to introduce a longitudinal field in that straight as shown in figure 3 .
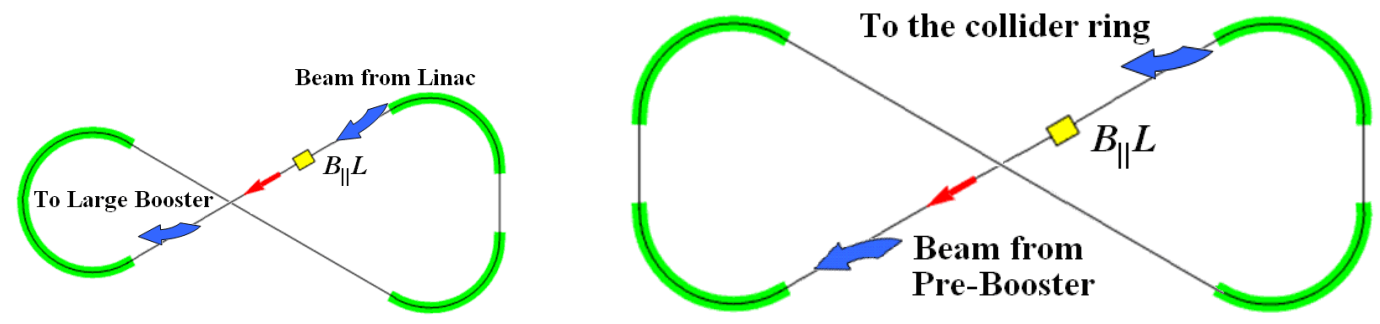

Figure 3: Matching of the polarization direction from the linac to the prebooster (left) and from the prebooster to the large booster (right).

The polarization is stable when the generalized spin tune $v$ determined by the longitudinal field is much greater than the strength of the zero-harmonic spin resonance $w_{0}$, i.e. when $v \gg w_{0}$. Table 1 gives parameters of the solenoids in the prebooster and large booster with the polarization directions matched at the beam injection and extraction locations. Note that, in a conventional accelerator with a Siberian snake, preserving the polarization during beam acceleration to $20 \mathrm{GeV}$ would require a "strong" solenoid with a field integral of $\sim 70 \mathrm{~T} \cdot \mathrm{m}$ for protons and of $\sim 230 \mathrm{~T} \cdot \mathrm{m}$ for deuterons.

Table 1: Parameters of the solenoids providing longitudinal polarization in the prebooster and large booster.

\begin{tabular}{l|c|c|c|c}
\hline & $p_{\text {inj }} / p_{\text {ext }}[\mathrm{GeV} / \mathrm{c}]$ & $\left(B_{\|} L_{\|}\right)_{\text {inj }} /\left(B_{\|} L_{\|}\right)_{\text {ext }}[\mathrm{T} \cdot \mathrm{m}]$ & $L_{\|}[\mathrm{cm}]$ & $v_{\text {deut }} / v_{\text {prot }}$ \\
\hline Prebooster & $0.785 / 3.83$ & $0.06 / 0.28$ & 60 & $0.003 / 0.01$ \\
\hline Large booster & $3.83 / 20$ & $0.28 / 1.5$ & 120 & $0.003 / 0.01$ \\
\hline
\end{tabular}

\section{Control of the deuteron polarization in the collider ring by small solenoids}

The deuteron polarization in the MEIC collider ring can be efficiently controlled by small solenoids. Any angle $\Psi$ lying in the orbit plane can be obtained between the polarization and the beam direction in an experimental straight by introducing longitudinal fields in two regions. Figure 4 shows a symmetric scheme of deuteron polarization control with two such insertions $(M=2)$ located on both sides of the experimental straight. The required spin rotation angles of the longitudinal fields in each insertion are given for small values of the spin tune $v$ by

$$
\varphi_{z 1}=\frac{2 \pi \nu}{M} \frac{\sin \left(\varphi_{y}-\Psi\right)}{\sin \varphi_{y}}, \quad \varphi_{z 2}=\frac{2 \pi \nu}{M} \frac{\sin \Psi}{\sin \varphi_{y}}
$$


where $\varphi_{z 1}=(1+G)\left(B_{\|} L\right)_{1} /(B \rho)$ and $\varphi_{z 2}=(1+G)\left(B_{\|} L\right)_{2} /(B \rho)$ are the spin rotation angles of the solenoids, $\varphi_{y}=\gamma G \alpha$ is the spin rotation angle of the dipole section between the solenoids, $\alpha$ is the orbit bending angle between the solenoids, $\Psi$ is the angle between the polarization and the beam direction, and $B \rho$ is the magnetic rigidity.

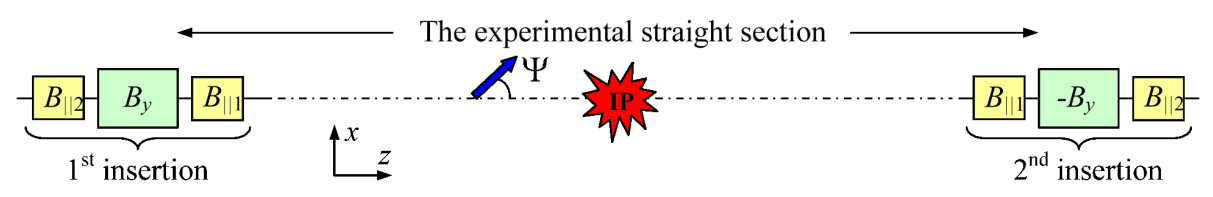

Figure 4: Deuteron polarization control scheme in the MEIC ion collider ring.

To work in the whole deuteron energy range in the collider, the first region with a longitudinal field integral $\left(B_{\|} L\right)_{1}$ is placed in the experimental straight while the second region with an integral $\left(B_{\|} L\right)_{2}$ is located after three regular dipoles with a total orbital bending angle of $13.2^{\circ}$ where there is a $21 \mathrm{~m}$ long straight in the MEIC collider lattice. The field integrals required in each insertion for obtaining the longitudinal $\left(\Psi=0^{\circ}\right)$ and transverse $\left(\Psi=90^{\circ}\right)$ polarizations are shown in figure 5 as functions of the beam momentum. The spin tune in the collider for the given field integrals has a value of $v=0.001$, which greatly exceeds the zeroth harmonic of the spin perturbations associated with imperfections of the collider's magnetic structure. The maximum field integral in a single solenoid at the maximum energy does not exceed $1.5 \mathrm{~T} \cdot \mathrm{m}$.

Thus, by choosing the necessary field integrals, the presented scheme allows one to obtain any polarization orientation in the collider's orbital plane at any orbital location, which can be used for matching the polarization at the beam injection as well as for obtaining the desired polarization in the second experimental straight. This also provides the capabilities of reversing the beam polarization during an experiment or changing it from the longitudinal to radial and vise versa.

$v_{D}=0.001, \varphi_{0 \mathrm{orb}}=13.2^{\circ}, \Psi=0^{\circ}$

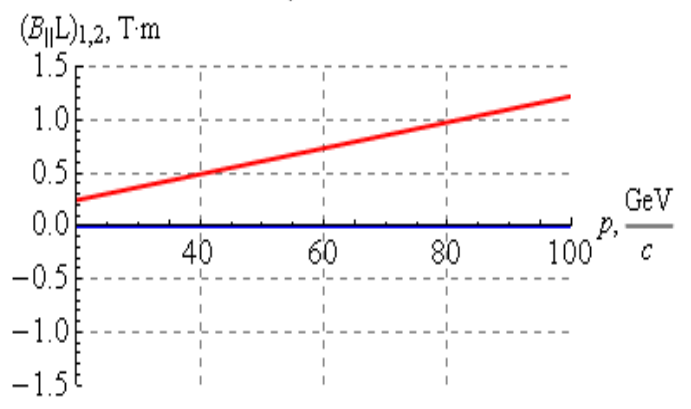

$\gamma_{D}=0.001, \varphi_{0 \mathrm{obb}}=13.2^{\circ}, \Psi=90^{\circ}$

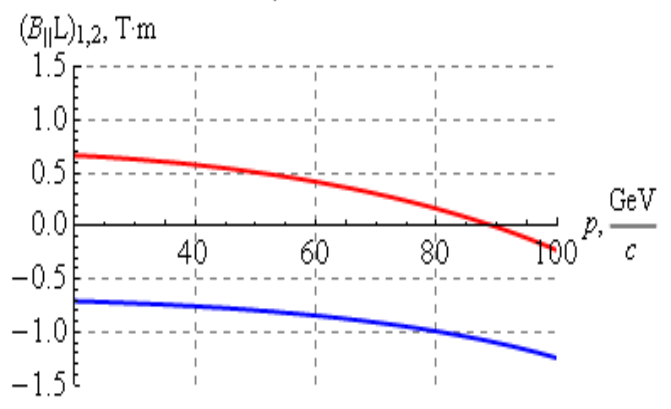

Figure 5: Dependence of the insertion's longitudinal field integrals $(\mathrm{T} \cdot \mathrm{m})$ on the beam momentum $(\mathrm{GeV} / \mathrm{c})$ for the longitudinal (left) and radial (right) polarizations of the deuteron beam in the MEIC collider ring. 


\section{Control of the proton polarization in the collider ring by small dipoles}

The scheme of deuteron polarization control with small solenoids is applicable to the proton beam as well. However, at high proton beam energies, instead of longitudinal fields, it is more suitable to use small radial fields, which are significantly lower than the longitudinal ones. The number of radial field regions in each insertion should be not less than four. This is due to the necessity of compensating the vertical orbit excursion caused by the radial dipoles. For the scheme to work in the whole proton beam energy range, the optimal bending angle of the arc dipoles is $\varphi_{\text {orb }}=0.82^{\circ}$. Figure 6 shows a symmetric scheme with two such insertions. The field integrals of the radial dipoles required to produce the spin tune of $v_{p}=0.01$ are shown in figure 7. The maximum radial field integral in a single dipole does not exceed $0.25 \mathrm{~T} \cdot \mathrm{m}$. Figure 8 shows the vertical orbit excursion inside the insertion containing the small dipoles at the injection and top MEIC collider energies.

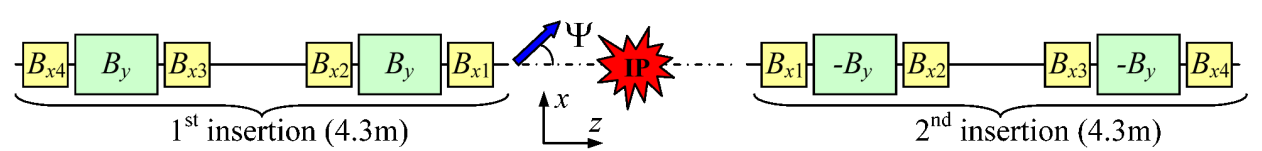

Figure 6: Scheme of proton polarization control using small radial fields.
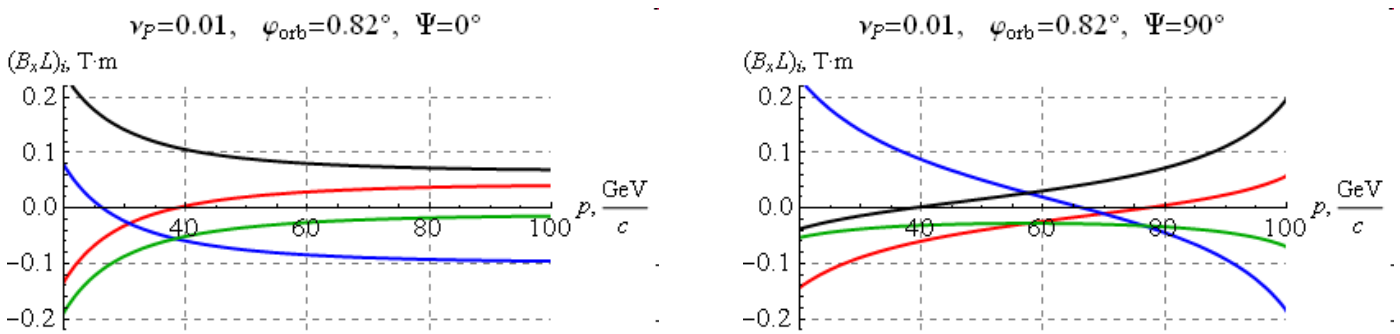

Figure 7: Dependence of the insertion's radial field integrals $(\mathrm{T} \cdot \mathrm{m})$ on the beam momentum $(\mathrm{GeV} / \mathrm{c})$ for the longitudinal (left) and radial (right) polarizations of the proton beam in the MEIC collider ring.

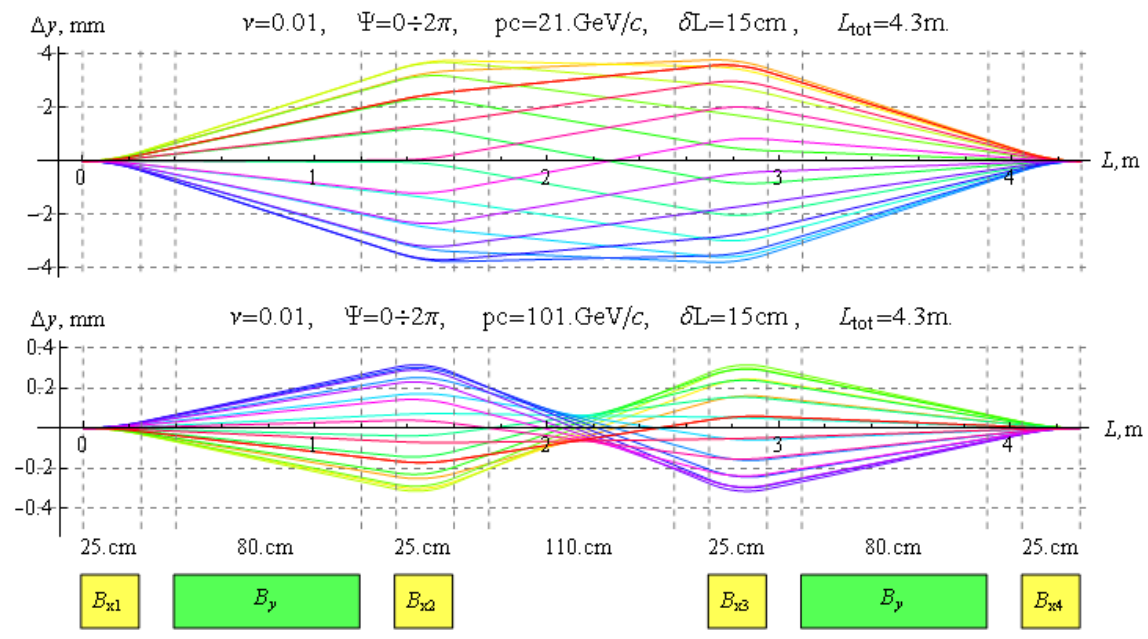

Figure 8: Vertical excursion of the reference orbit at the injection and top MEIC collider ring energies. 


\section{Response function and zero-harmonic spin resonance strength in the collider ring}

The strength of the zero-harmonic spin resonance $w_{0}$ is determined by the vertical excursions of particle trajectories from the collider's plane. At high energies, the greatest contribution to the strength of this resonance comes from the radial magnetic field components on the particle trajectories. In practice, the vertical excursion of the beam's closed orbit due to imperfections of the collider's magnetic structure substantially exceeds the beam size. Therefore, dependence of the strength $w_{0}$ on the beam emittances is much less significant.

The coherent part of the spin resonance strength can be calculated using a spin response function. The periodic response function $F(\theta)$ is determined by the accelerator's ideal magnetic structure and is a response to a perturbing radial field $b_{x}(\theta)$ at an azimuthal angle $\theta$ $(z=R \theta)$ :

$$
w_{0}=\frac{G \gamma R}{2 \pi B \rho} \int_{0}^{2 \pi} b_{x} F(\theta) d \theta
$$

As an example, figure 9 shows a graph of the response function for a deuteron beam at the top energy in the MEIC collider ring. The graph indicates that the main contribution to the resonance strength comes from the interaction regions. Hence, it is important to use an appropriate magnetic structure near the interaction regions to compensate the impact of lattice imperfections in the experimental straights.

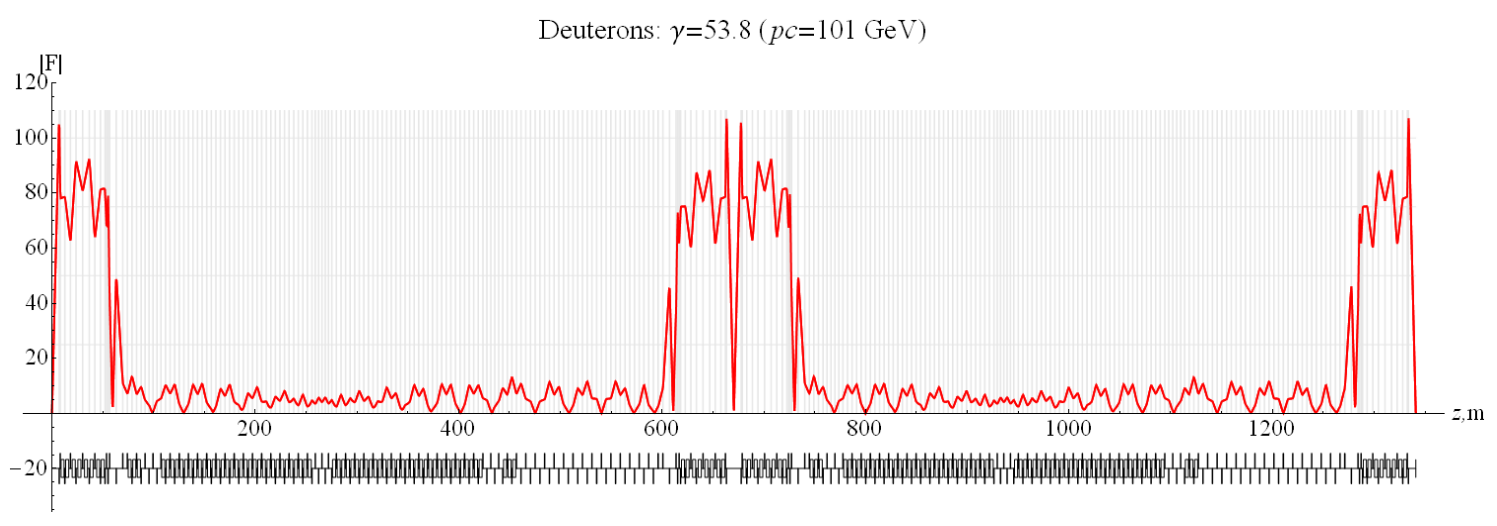

Figure 9: Response function for a deuteron beam in the MEIC collider ring.

The coherent part of the resonance strength $w_{0}$ can be compensated by corrector magnets. In the linear approximation, the strength $w_{0}$ is determined by two components of the spin perturbation lying in the collider's plane and can be compensated by correcting devices rotating the spin about an axis lying in the same plane. The role of such devices can be played by the insertions with two solenoids or with four radial dipoles described above in the context of polarization control.

Our estimates indicate that, after compensation of the coherent part of the zero-harmonic spin resonance strength, the strength due to the beam emittances is of the order of $10^{-3}$ for 
protons and $10^{-4}$ for deuterons. In the next stage, compensation of the incoherent part of $w_{0}$ requires optimization of the magnetic structure's focusing properties.

There is a unique opportunity of building an "ideal" collider with polarized beams using a real magnetic structure. The polarization orientation can be controlled by ultimately-small magnetic field integrals. This will allow polarized beam experiments with the highest precision levels. Such a tool can detect the slightest polarization changes on long time scales.

\section{Summary}

To summarize, let us briefly state the main conclusions. Polarization schemes have been developed for the MEIC figure- 8 rings that (i) virtually eliminate the depolarization problem during acceleration, (ii) allow control of the beam polarization using small fields without significant orbit perturbation, (iii) make it possible to efficiently control the polarization of a beam of particles with any anomalous magnetic moment including those with a small one, such as deuterons, (iv) allow adjustment of any polarization orientation in either of the two straights, (v) allow single-turn and multi-turn spin-flipping schemes [3], and (vi) make possible ultra-high precision experiments with polarized beams.

\section{Acknowledgements}

Authored by Jefferson Science Associates, LLC under U.S. DOE Contract No. DE-AC0506OR23177 and DE-AC02-06CH11357. The U.S. Government retains a non-exclusive, paidup, irrevocable, world-wide license to publish or reproduce this manuscript for U.S. Government purposes.

\section{References}

[1] S. Abeyratne et al., Science requirements and conceptual design for a polarized medium energy electron-ion collider at Jefferson lab, edited by Y. Zhang and J. Bisognano, arXiv:1209.0757 [physics.acc-ph] (2012).

[2] A.M. Kondratenko et al., Preservation and control of the proton and deuteron polarizations in the proposed electron-ion collider at Jefferson lab, to be published in proceedings of $20^{\text {th }}$ International Spin Physics Symposium (SPIN 2012), Dubna, Russia, September 2012.

[3] A.M. Kondratenko et al., Spin-flipping systems for storage rings, in proceedings of the $14^{\text {th }}$ Advanced Research Workshop on High Energy Spin Physics (DSPIN-11), Dubna, Russia, September 2011, edited by A.V. Efremov and S.V. Goloskokov (JINR, Dubna, Russia, 2012), p. 377. 